\title{
Prp4 kinase is required for proper segregation of chromosomes during meiosis in Schizosaccharomyces pombe*
}

\author{
Miroslava Pozgajova ${ }^{\boxplus}$, Lubos Cipak² and Anna Trakovicka \\ 'Slovak University of Agriculture, Department of Genetics and Breeding Biology, Nitra, Slovakia; ${ }^{2}$ Cancer Research Institute, Slovak Academy of \\ Sciences, Bratislava, Slovakia
}

\begin{abstract}
Chromosome segregation during meiosis is a complex process, which leads to production of four haploid gametes from two precursor cells. Reversible phosphorylation of proteins plays a crucial role in this process. The Schizosaccharomyces pombe Prp4 is an essential serine/ threonine protein kinase, which belongs to the Clk/Sty family. To study the role of Prp4 in meiosis, we analysed chromosome segregation in a strain carrying conditional analog-sensitive allele of Prp4 protein kinase (prp4-as2). Our data show, that Prp4 protein kinase plays important role in chromosome segregation during meiosis, as revealed by enhanced missegregation of chromosomes in prp4-as2 mutant cells.
\end{abstract}

Key words: Prp4 protein kinase, S. pombe, meiosis, segregation, protein phosphorylation

Received: 15 October, 2013; revised: 03 December, 2013; accepted: 18 December, 2013; available on-line: 29 December, 2013

\section{INTRODUCTION}

The basic feature of all living organisms is cell division, which is required for growth and development. The cell undergoes various signal-transduction processes, to achieve its proper division. Protein phosphorylation by kinases is one of the key mechanisms required for regulation and maintenance of the normal cell cycle (Krebs, 1993). Ortholog of the essential S. pombe protein kinase Prp4 was previously described to play important role in chromosome alignment, and is involved in spindle assembly checkpoint (SAC) regulation. (Montembault et al., 2007). Moreover, it is a serine/threonine kinase, which was shown to regulate pre-mRNA splicing in mammalian cells (Rosenberg et al., 1991; Schneider et al., 2010). However, its role in chromosome segregation during meiosis was not yet described. Therefore, we prepared strain carrying conditional analog-sensitive allele of Prp4 (prp4-as2(F238A)), where the gate-keeper residue within an ATP binding site of the Prp4 kinase (F238) was mutated to induce its affinity to the inhibitor (Cipak et al., 2011; Gregan et al., 2007). In this study, we analysed such strain in the presence or absence of the inhibitor, respectively, and compared it to wild type strain.

Our data revealed that genetic manipulations of the Prp4 kinase cause significant alterations in the process of chromosome segregation, even in the absence of the inhibitor as compared to wild type strain. The presence of the inhibitor enhanced the already existing defect, showing that the inhibitor blocked remaining, still functional protein kinase, and confirmed, that Prp4 protein kinase plays important role in the process of chromosome segregation.

\section{MATERIALS AND METHODS}

Yeast strains, media and growth conditions. $S$. pombe strains used to test sensitivity to ATP analog (3-BrB-PP1) were wild type (b-) and prp4-as2 (b-leu1-32 ura4-D18 ade6-M210 prp4::natMX4 prp4-as2(F238A)::bphMX4) strains. Sequence analysis of our prp 4 -as 2 strain revealed the presence of both wild-type $p r p 4^{+}$and the prp4-as2(F238A) alleles, raising the possibility that this strain expresses a wild-type Prp4 protein in addition to Prp4-as2 (unpublished data). Yeast media and growth conditions were standard YE+5S or PMG-N media in the presence or absence of an ATP analog (Cipak et al., 2011).

Immunostaining and microscopy. The immunofluorescence and microscopy techniques used to analyze chromosome segregation were as was previously described. Shortly, yeast strains with or without chromosome II labeled with GFP (cen2(D107)::KanR-ura4+-lacO bis7+.:lacI-GFP) were grown in YE $+5 \mathrm{~S}$ medium and meiosis was induced by crossing $h+$ and $h-$ strains on PMG$\mathrm{N}$ plates. After conjugation, strains were transferred to liquid PMG-N medium and inhibitor or DMSO were added. Cells were collected after 7 hours of incubation, fixed, and stained with primary TAT1 mouse monoclonal anti-tubulin and rabbit polyclonal anti-GFP antibodies, DNA was visualised using Hoechst 33342 (Rabitsch et al., 2004; Rumpf et al., 2010). Analyses were performed using regular fluorescent microscope equipped with digital camera.

\section{RESULTS}

To study the role of $S$. pombe essential Prp4 protein kinase during meiosis, we used prp4-as 2 strain and analysed its chromosome segregation during meiosis in the presence or absence of the inhibitor and compared it to the wild type $(w t)$ strain.

\section{Analysis of sensitivity of prp4-as2 to the inhibitor}

The sensitivity of $w t$ and prp4-as2 strains to the inhibitor was tested on YES plates with or without addition of $30 \mu \mathrm{M}$ of the inhibitor (3-BrB-PP1). Cells were

e-mail: miroslava.pozgajova@uniag.sk

*Presented at the 5th Central European Congress of Life Sciences „EUROBIOTECH 2013”, Kraków, Poland. 


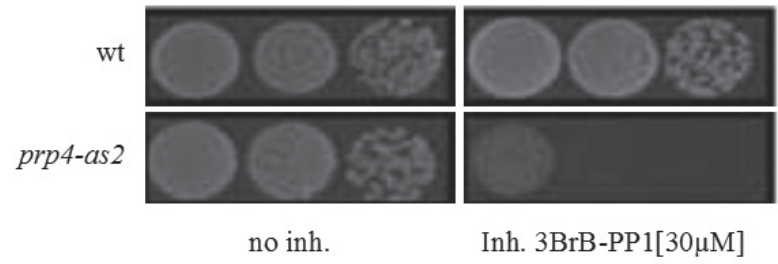

Figure1. Sensitivity of prp4-as2 cells to the inhibitor. Prp4-as2 cells were able to grow on YES plates lacking the inhibitor, whereas in the presence of the inhibitor cell growth was inhibited. However, wt strains were able to grow under both conditions, pointing-out the effect of the inhibitor only to mutated strains (Cipak et al. 2011)

spotted on plates, incubated at $32^{\circ} \mathrm{C}$ for $3-4$ days, and their growth ability was determined. As shown on Fig. 1, prp4-as2 cells grew on YES plates lacking the inhibitor, whereas in the presence of the inhibitor cell growth was inhibited. However, wt strain was able to grow under both conditions, pointing-out the effect of the inhibitor only to mutated strain (Cipak et al. 2011).

\section{Chromosome segregation during meiosis is altered in prp4-as2 cells}

To analyze chromosome segregation directly in anaphase I, cells with one copy of chromosome II marked by cen2-GFP were fixed and stained with antibodies against tubulin and GFP. DNA was stained with Hoechst 33342. Chromosome segregation was analysed in wt and prp4-as 2 cells incubated for 7 hours with the inhibitor or DMSO respectively using the fluorescent microscope. We observed large amount of lagging chromosomes in prp4-as2 cells even in the absence of the inhibitor $(12.5 \%$ of analysed cells), indicating, that already small manipulation with the ATP binding side of Prp4 protein kinase causes alterations in its function. Addition of the inhibitor doubled the observed defect suggesting, that remaining, still functional Prp4 protein kinase was blocked with the use of the inhibitor. Moreover, defects in segregation of sister chromatids (visualized by heterozygous cen2-GFP) during meiosis I in prp4-as2 cells were also observed already in the absence of the inhibitor $(6.25 \%$ of analysed cells showed missegregation), and the observed defect was enhanced when the inhibitor was added (17.6\% of analysed cells). (Fig. 2) These results suggest that Prp4 protein kinase is involved in regulation of the first meiotic division, and thereby controls the process of correct chromosome segregation.

\section{DISCUSSION}

Protein phosphorylation has been established as the major regulatory mechanism in the cell. Some protein kinases are already known to be involved in meiotic chromosome segregation, such as Hhp1/Hhp2, Bub1, Ark1, and Mph1 (Rabitsch et al., 2004; Rumpf et al., 2010; Kovacikova et al., 2013). In order to identify novel protein kinases involved in regulation of chromosome segregation during meiosis, we screened a collection of our analog-sensitive essential protein kinases for their role in meiosis (Cipak et al., 2011; unpublished results). It was previously shown that Prp4 is important for pre-mRNA splicing in human and yeast cells (Gross et al., 1997; Schneider et al., 2010), but it is also component of the phosphoproteome in mitotic spindle which contains kinetochore-associated proteins

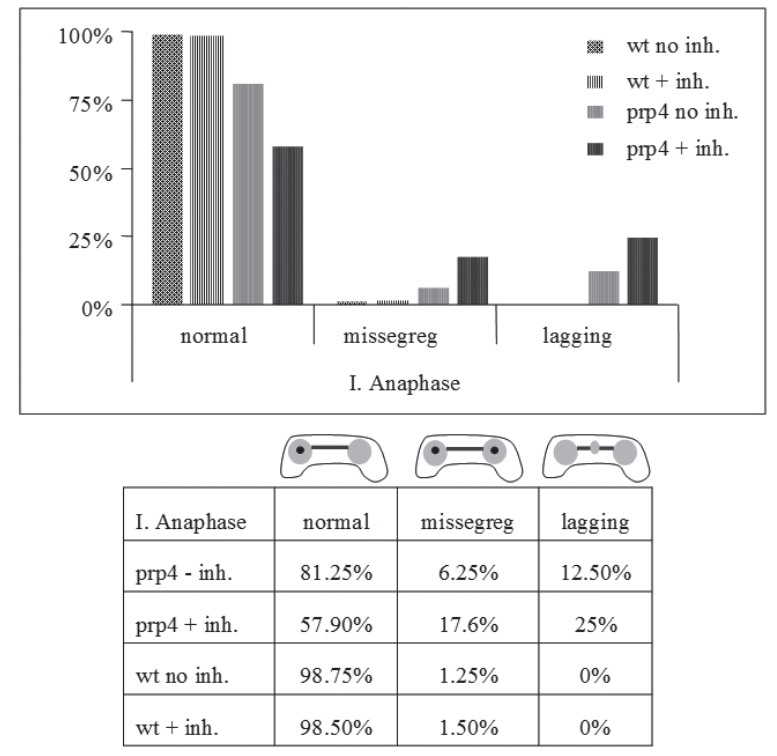

Figure 2. Prp4 is required for proper chromosome segregation during meiosis.

Missegregation in prp4-as2 cells (prp4) with high amount of lagging chromosomes during anaphase I indicates defect in segregation of sister chromatids (only one copy of chromosome II was labelled with cen2-GFP).

in human cells (Nousiainen et al., 2006) and part of SAC (spindle assembly checkpoint) regulatory genes (Montembault et al., 2007). However, direct analysis of the function of Prp4 protein kinase in chromosome segregation was not performed so far as it is an essential protein and lack of its function is lethal for the cell. Therefore, we used a conditional mutant form of Prp4 protein kinase (prp4-as2(F238A)) that is inhibited in the presence of ATP analog, to directly investigate its role in meiotic chromosome segregation. Strikingly, our analyses revealed, that inhibition of Prp4 protein kinase causes massive defects in chromosome segregation during meiosis. While missegregation of sister chromatids and presence of lagging chromosomes during meiosis I can be caused by various defects, such as defective sister-chromatid cohesion along chromosome arms (Gutierrez-Caballero et al., 2012), it is very likely, that the observed defect in prp4-as 2 cells is probably due to alterations in spindle-kinetochore attachments in anaphase I. This is in agreement with previously published results, that Prp4 is kinetochore component required for functional SAC (Montembault et al., 2007).

We identified this essential protein kinase as a novel protein kinase required for regulation of proper chromosome segregation during meiosis, as revealed by enhanced missegregation of sister chromatids, and enhanced occurrence of lagging chromosomes in prp4-as2 mutant cells. However, further investigations are required to analyse the underlying mechanism of Prp4 function in this process.

\section{Acknowledgements}

This work was financially supported by the Slovak Research and Development Agency under the Contract No. APVV-0636-11 and APVV-0111-12 and by Seventh Framework Programme (FP7/2007-2013) under grant 
agreement number PERG07-GA-2010-268167 and by Austrian Science Fund grants P23609 and P21437.

\section{REFERENCES}

Cipak L, Zhang C, Kovacikova I, Rumpf C, Miadokova E, Shokat KM, Gregan J (2011) Generation of a set of conditional analog-sensitive alleles of essential protein kinases in the fission yeast Schizosaccharomyces pombe. Cell Cycle 10: 3527-3532.

Gregan J, Zhang C, Rumpf C, Cipak L, Li Z, Uluocak P, Nasmyth K, Shokat KM (2007) Construction of conditional analog-sensitive kinase alleles in the fission yeast Schizosaccharomyces pombe. Nat Protoc 2: 2996-3000.

Gross T, Lutzelberger M, Weigmann H, Klingenhoff A, Shenoy S, Kaufer NF (1997) Functional analysis of the fission yeast Prp4 protein kinase involved in pre-mRNA splicing and isolation of a putative mammalian homologue. Nucleic Acids Res 25: 1028-1035.

Gutierrez-Caballero C, Cebollero LR, Pendas AM (2012) Shugoshins: from protectors of cohesion to versatile adaptors at the centromere. Trends Genet 28: 351-360.

Kovacikova I, Polakova S, Benko Z, Cipak L, Zhang L, Rumpf C, Miadokova E, Gregan J (2013) A knockout screen for protein kinases required for the proper meiotic segregation of chromosomes in the fission yeast Schizosaccharomyces pombe. Cell Cycle 12: 618-624.
Krebs EG (1993) Nobel Lecture. Protein phosphorylation and cellular regulation I. Biosci Rep 13: 127-142.

Nousiainen M, Sillje HH, Sauer G, Nigg EA, Korner R (2006) Phosphoproteome analysis of the human mitotic spindle. Proc Natl Acad Sci USA 103: 5391-5396.

Montembault E, Dutertre S, Prigent C, Giet R (2007) PRP4 is a spindle assembly checkpoint protein required for MPS1, MAD1, and MAD2 localization to the kinetochores. I Cell Biol 179: 601-609.

Rabitsch KP, Gregan J, Schleiffer A, Javerzat JP, Eisenhaber F, Nasmyth K (2004) Two fission yeast homologs of Drosophila Mei-S332 are required for chromosome segregation during meiosis I and II. Curr Biol 14: 287-301.

Rosenberg GH, Alahari SK, Kaufer NF (1991) Prp4 from Schizosaccharomyces pombe, a mutant deficient in pre-mRNA splicing isolated using genes containing artificial introns. Mol Gen Genet 226: 305-309.

Rumpf C, Cipak L, Dudas A, Benko Z, Pozgajova M, Riedel CG, Ammerer G, Mechtler K, Gregan J (2010) Casein kinase 1 is required for efficient removal of Rec8 during meiosis I. Cell Cycle 9: 26572662.

Schneider M, Hsiao H, Will CL, Giet R, Urlaub H, Lührmann R (2010) Human PRP4 kinase is required for stable tri-snRNP association during spliceosomal B complex formation. Nat Struct Mol Biol 17: 216-221. 